\title{
Evidence of muscle synergies during human grasping
}

\author{
Claudio Castellini • Patrick van der Smagt
}

Received: 25 April 2012 / Accepted: 12 January 2013 / Published online: 31 January 2013

(C) Springer-Verlag Berlin Heidelberg 2013

\begin{abstract}
Motor synergies have been investigated since the 1980s as a simplifying representation of motor control by the nervous system. This way of representing finger positional data is in particular useful to represent the kinematics of the human hand. Whereas, so far, the focus has been on kinematic synergies, that is common patterns in the motion of the hand and fingers, we hereby also investigate their force aspects, evaluated through surface electromyography (sEMG). We especially show that force-related motor synergies exist, i.e. that muscle activation during grasping, as described by the sEMG signal, can be grouped synergistically; that these synergies are largely comparable to one another across human subjects notwithstanding the disturbances and inaccuracies typical of sEMG; and that they are physiologically feasible representations of muscular activity during grasping. Potential applications of this work include force control of mechanical hands, especially when many degrees of freedom must be simultaneously controlled.
\end{abstract}

Keywords Rehabilitation robotics · Grasping ·

Electromyography

\section{Introduction}

The human hand has a rather complex biomechanical structure, and a to date not completely understood neural archi-

C. Castellini $(\square)$

DLR / German Aerospace Center, Institute of Robotics and Mechatronics, Muenchnerstr. 20, 82234 Wessling, Germany e-mail: claudio.castellini@dlr.de

\section{P. van der Smagt}

Institute of Computer Science VI Technische Universität München, Boltzmannstr.3, 85748 Garching, Germany

e-mail: smagt@tum.de tecture to control it. In the analysis of the biomechanical and behavioural aspects of the hand, one of the most striking aspects is the high redundancy of its structure, seemingly having many more degrees of freedom than are actually used/required. In order to cope with this apparent redundancy, the concept of synergies has been used to describe functional dependencies among degrees of freedom. Bernstein (1967) defines the level responsible for coordinating large muscle groups and different movement patterns as the level of muscular-articular links or synergies. Thus, the state space of the system can be reduced to a reduced number of independent dimensions. A similar finding is the well-known combination of motor primitives in frogs by Mussa-Ivaldi et al. (1994).

A number of recent experimental studies confirm this theory for the human hand, too. As shown by Santello et al. (1998, 2002), the simultaneous motion of the fingers is characterised by coordination and covariation patterns that reduce the number of independent degrees of freedom to be controlled by the nervous system. Still, although some constraints on the musculotendon system, as well as on the peripheral and central nervous system, can be identified, a clear relationship between the finger kinematic constraints and the underlying muscular activity remains to be analysed. As a matter of fact, the source of such kinematic synergies in the human hand remains a matter of debate; indeed, the biomechanical structure of the hand, in which tendons activate multiple digits at the same time, while the related muscles share common bases, is one source for the synergies (see, e.g. (Lang and Schieber 2004)); but the spinal circuitry, mapped only to a small extent to the human hand, co-activates muscles and thus defines synergies (Takei and Seki 2010); and at the highest level, cortical organisation (Holdefer and Miller 2002) is suspected to play a dominant but variable role in these. 
We are hereby concerned with the expression of force synergies in the human hand, when placed in a real-life environment, i.e. in our case, grasping. When an object is grasped/held/manipulated, the relationship between hand kinematics and the forces involved becomes much more complex; to make a detailed analysis, one should use a detailed model of the environment, of the involved objects and of the musculoskeletal system to reconstruct the forces from the kinematic data. The other side of this issue is represented by impedance control of robotic (possibly prosthetic) hands: impedance-based control schemes lead to a much higher control stability, especially in the case where physical contact is involved (Grebenstein and Smagt 2008; Arbib et al. 2008). But, the generally high number of degrees of freedom of a poly-articulated, multi-fingered mechanical hand requires at least a coordination principle. It is likely that a few simultaneous and coordinated forces can be produced and then mapped onto the degrees of freedom involved without losing too much dexterity; as kinematic synergies do exist in the control of the human hand, it seems reasonable to believe that analogous synergies at the level of muscle activation should be found as well.

We hereby use surface electromyography (sEMG) to detect muscular activation in the forearm during a grasping task in humans, and we then check whether a similar simplifying principle (which we will call muscle synergies from now on) can be found. Muscular activity measured via sEMG relates in the simplest case nearly linearly to the force exerted by a muscle (De Luca 1997, 2002); but, given the complexity of the arm/forearm/hand musculoskeletal system, there is no a priori indication as to whether such a principle should exist although finger position synergies have been found in Santello et al. (1998). Some results mapping sEMG activity to finger, wrist and arm position have actually appeared, e.g. in (Tsuji et al. 1993; Tenore et al. 2009; Vogel et al. 2011), but the assumption there is that sEMG relates to isotonic/isometric muscle configurations which, in free movement, can roughly be associated to positions. Here, we are concerned with grasping, therefore those results are not relevant to this work; when dynamic interaction with the environment (e.g. objects to be grasped) comes into play, any such trivial relationship is likely to be broken down.

In this paper, we show that muscle synergies do exist. We describe an experiment in which 5 human subjects would grasp 5 objects in 5 accordingly different ways, and show that there is considerable statistical overlapping among muscle synergies found across the subjects. In other words, all subjects seem to enact a certain grasp by activating the same muscles (or groups of motor units) all over. Muscular activity is gathered using sEMG via 10 commercial prosthetic electrodes, placed on the forearm of each subject without targeting any particular muscle. (This qualifies our setup as simple, easy to use, which is a plus when thinking of possible application of this research.) The fact that common human muscle synergies can be identified, notwithstanding the wellknown problems associated to sEMG (muscle cross-talk, sweating, inter-subject anatomical differences, inaccurate electrode positioning and so on) paves the way to synergistic force- or impedance-based control of robotic hands. Once muscle synergies are identified for a subject, they could constitute high-level force commands for the hand, which would then be mapped back onto the single degrees of freedom of the hand itself, therefore greatly simplifying its control. (A promising result along this line of research appears, e.g. in (Wimböck et al. 2011).)

A further qualitative analysis of the muscle synergies and of how the grasps map onto them reveals some regularities that can be actually mapped onto the anatomy and dynamics of the forearm, making this result interesting from the point of view of physiology, too. A similar line of research has been so far pursued, as far as we know, only in primates and only loosely in humans. Primates have been shown to generate consistent-across-subject sEMG patterns in Brochier et al. (2004); Overduin et al. (2008) when engaged in simple grasping actions; in those works up to 19 needle (invasive), sEMG electrodes were used on (in both papers) two animals. In particular, in Overduin et al. (2008), it was shown that three synergies accounted for $81 \%$ of the sEMG variance, but the analysis performed was time dependent, meaning that synergies are short temporal profiles of activation rather than single sEMG samples. In this work, we concentrate on a simpler PCA-based dimensionality reduction (which is the type effectively used in Brochier et al. (2004) as well as, e.g. in (Santello et al. 2002)) and obtain quantitatively similar results, that is, as far as the amount of variance is concerned.

Human beings have been studied from this point of view, e.g. in (Grinyagin et al. 2005), where a detailed musculoskeletal model was used to reconstruct finger joint torques from kinematic data (obtained with a Cyberglove similar to ours). In this case too, similarity among grip patterns was found. Of course, this analysis cannot be applied to grasping since joint torques in the latter case depend on the objects too.

The work we present here is novel in that, as far as we know, no detailed analysis of sEMG patterns during grasping (their inter-subject similarity, repeatability, stability and anatomical relevance) has been so far attempted on humans. We could afford such an analysis since we use surface electromyography, which is cheap, non invasive and easy to use - but, it cannot bear detailed information about single muscles, let alone single motor units, although some work on sEMG decomposition (Hamid Nawab et al. 2008) points to that direction.

The paper is organised as follows: we describe the experiment in Sect. 2, results are presented in Sect. 3 and conclusions are drawn in Sect.4. This paper can be seen as the 


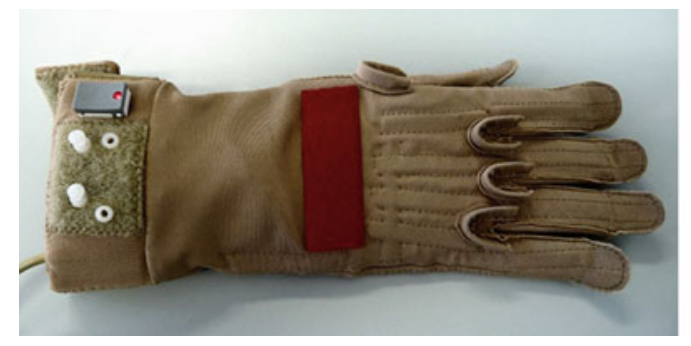

Fig. 1 Data capturing devices and sEMG electrode arrangement. (left to right) the Cyberglove we used, an 18-sensor model; schematic representation of the location of the sensors on the Cyberglove (22-sensors
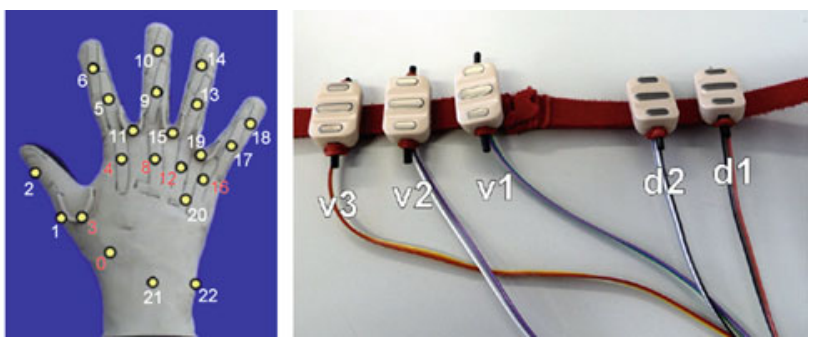

model); 5 sEMG electrodes arranged with rubber bands on a Velcro strap; labels denote ventral (v1, v2, v3) and dorsal (d1, d2) electrodes. natural followup to Castellini and van der Smagt (2011), where a much less detailed analysis was carried out, and no analysis of physiological relevance appeared.

\section{Experiment description}

\subsection{Data gathering}

\subsubsection{Hand motion}

An 18-sensor right-handed Cyberglove (Cyberglove Systems, www.cyberglovesystems.com, see also Fig. 1, left panel) was used to gather the finger positions. The Cyberglove is a light, fabric, rather elastic glove, onto which 18 strain gauges are sewn; the sewing sheaths are chosen carefully by the manufacturer so that the gauges exhibit a resistance which is proportionally related to the angles between pairs of hand joints of interest. The device returns 18 8-bit values proportional to these angles, having a resolution of less than one degree. The resolution is declared to slightly change accordingly to the size of the subject's hand, careful wearing and the rotation range of the considered joint. (For practical reasons the subject must wear a friction-reducing glove below the Cyberglove; an initial round of data gathering revealed that this would not limit the precision of the device.) We hereby consider all sensor values of the glove, that is, 18 8 -bit values. Figure 1, centre panel shows the placement of the sensors on the 22-sensors variant of the glove, which has 4 additional sensors at the distal phalanges.

\subsubsection{Surface electromyography}

Muscular activity was gathered by ten Otto Bock MyoBock 13E200 active, double-differential sEMG electrodes (www. ottobock.com). These electrodes process the raw sEMG signal using an on-board amplification/bandpass-filtering/rectifying circuit. In the typical case, when they are applied over a single, large muscle, the resulting output is quasi-linearly related to the force exerted by the muscle, or rather, to the percentage of the maximum voluntary contraction of the muscle (see De Luca (1997, 2002); in Zecca et al. (2002), the raw signal is visible in Fig. 2(a), whereas the signal obtained from these electrodes is similar to that in Fig. 2(c)). The use of this signal rather than the raw one is preferred here since digital processing is considerably simplified; the unavoidable delay of around $200 \mathrm{~ms}$ introduced by filtering can be neglected for the purpose of our study due to the chosen data processing (see below). The usefulness of this kind of electrodes, and of the signal they provide, has already been demonstrated at least in Castellini and van der Smagt (2009); Castellini et al. (2009).

The electrodes were placed on the subject's forearm surface according to the following rules: they were split in two sets of five, each set arranged in two lines of two plus three electrodes, firmly tied to a Velcro strap using elastic bands (see Fig. 1, right panel). The bands and straps were tied around the forearm so that

1. the first band surrounded the forearm about $5 \mathrm{~cm}$ below the elbow,

2. the second band surrounded the forearm midway between the elbow and the wrist,

3. the groups of two electrodes would lie on the dorsal side of the forearm, whereas the groups of three would lie on the ventral side.

This placement is intentionally largely irrespective of the (internal) anatomy of the human forearm so that no medical consultancy is required (no search for relevant muscles is performed before the straps are secured). Uniform placement, irrespective of anatomy, has already been demonstrated effective, even on amputees (Castellini et al. 2009), for sEMG signals classification and regression purposes. In the following, we will refer to the electrodes below the elbow as to the proximal electrodes, to those midway on the forearm as to the distal electrodes and to the electrodes in general as ventral or dorsal according to the side of the forearm they were placed 
upon. Ventral electrodes are denoted v1, v2 and v3, whereas dorsals are denoted d1, d2 (Figs. 1 (right panel) and 2).

The electrodes are connected to a DAQ card sampling the sEMG signals at $100 \mathrm{~Hz}$.

\subsection{Experimental protocol}

Six healthy human subjects (all male, all right-handed, age $24 \div 45$ years, $32 \pm 8.2$ years) joined the experiment; informed consent was obtained from them before the experiment. The subjects would sit comfortably in front of a desk; their right

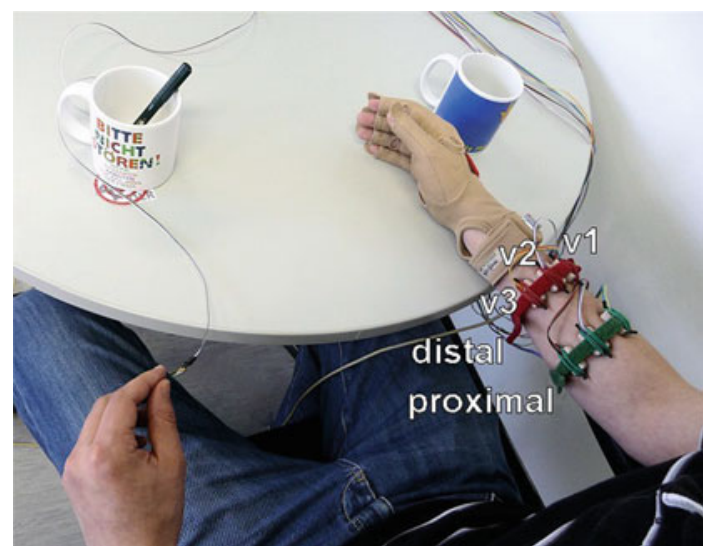

Fig. 2 Bird's eye view of the experimental setup; the proximal and distal ventral electrodes (v1, v2 and v3) are clearly visible, fixed on the subject's right forearm. (The Figure also depicts a pressure sensor held with the left hand which was not used in the data processing.) hand and forearm would be fitted with the electrode sets and the glove; they would then be instructed to put the right elbow on the chair's armrest, and the right hand on the table; finally, they were instructed to relax. The forearm was kept in a halfpronated posture, such that its ventral side would be parallel to the sagittal plane, as if to grasp cylindrically an object (Fig. 2).

Two spots on the table were highlighted by a clearly visible marker; then, under the strict request not to rotate the forearm, the subjects would be instructed to reach and grasp an object placed onto the desk over the first marked spot; to carry it over to the second spot; to drop it over there; and finally to go back to the resting position. (Each object was lying in such a position that it would be comfortably grasped without rotating the forearm.) The operation was indifferently performed this way, or from the second to the first spot. When the object could not be easily laid standing, two mugs were used to drop it inside them. This way, a reach/grasp/carry/drop/rest sequence was performed.

The requirement to avoid lower arm rotation was due to the necessity of keeping as much as possible constant the position of the electrodes with respect to the muscles of the forearm. It is easy to ascertain by palpation, actually, that the forearm skin moves dramatically with respect to the muscles, when pronating/supinating. Such an uncontrolled movement would have probably introduced too much noise in the gathered data. The experimenter took care that the pronation/supination movement was actually never performed during the experiment.

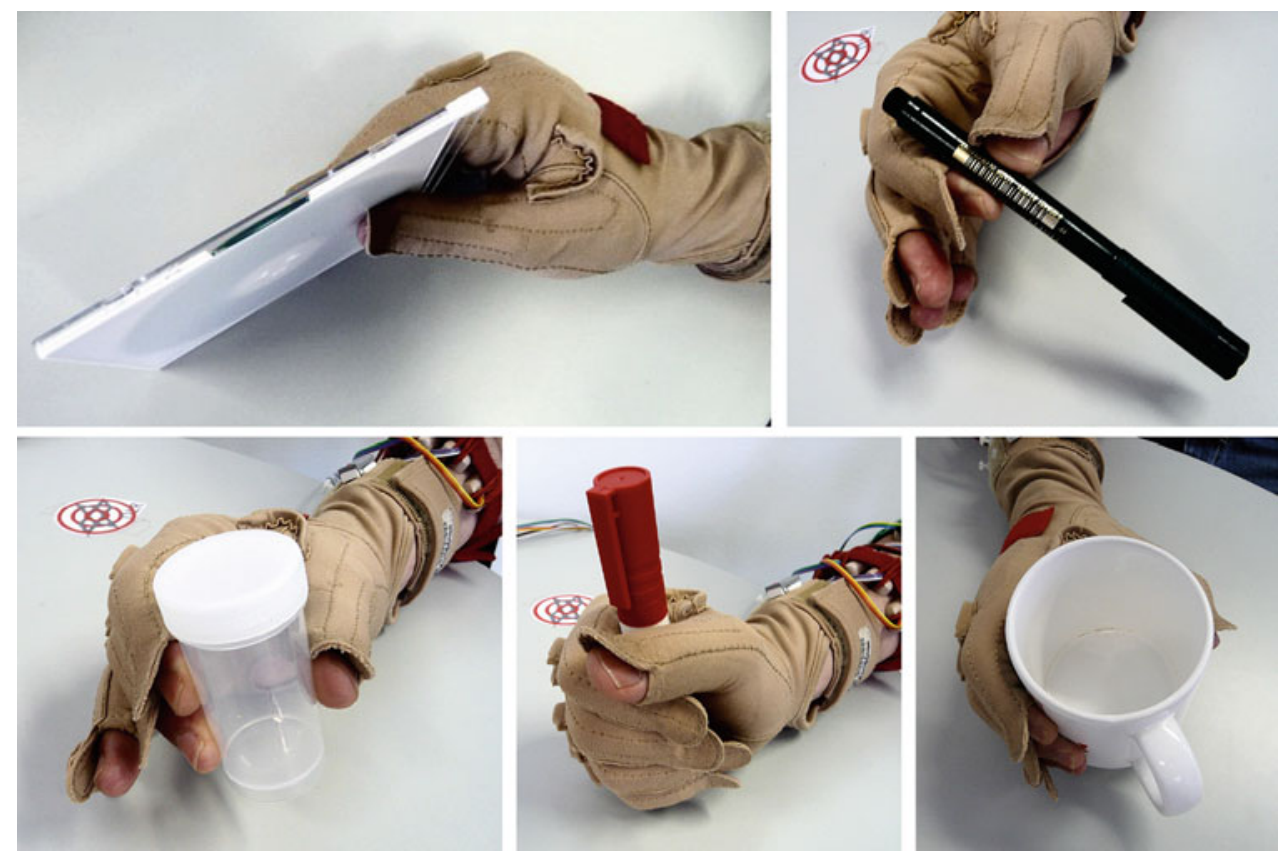

Fig. 3 The five objects while being grasped by a subject: (left to right) flat grasp, pinch grip, tripodal grip, small power grasp and large power grasp. Notice that the subject never pronates and/or supinates the forearm, as instructed 
The above-described sequence was repeated for 20 times for each object; 5 objects were in turn used, each one to be grasped in a different way. Since 6 subjects joined in the experiment, it was expected that at the end 600 sequences would be gathered. The objects and grasps selected were a DVD (to be grasped with a flat grasp), a pen (pinch grip), a small plastic container (tripodal grip), a dry wipe marker (small power grasp) and a mug (large power grasp). Figure 3 depicts the objects and the ways to grasp them. The movie 'grasping.avi' provided as supplemental material shows some typical grasping sequences performed by a subject; in the movie, the carrying phase, that is the phase during which the object would be held firmly and carried from one spot to the other, is clearly indicated. The carrying phase is the time interval of interest, since it is assumed that during it, a stable grasp configuration would be achieved. The experimenter verified visually that that would be the case in all grasps considered; the fact that (Fig. 1, left panel again) the Cyberglove we used has no fingertips proved here to be very useful, as it allowed the subject retain full friction at the finger pads, allowing for completely natural grasps.

Each sequence lasted less than $3 \mathrm{~min}$ for a total of about $15 \mathrm{~min}$ for each subject. No subjects reported fatigue, discomfort or pain during or after the experiment.

\subsection{Data synchronisation and preprocessing}

Data synchronisation was enforced on a Windows PC by gathering data from each device asynchronously and accurately time stamping each received datum. Time stamping was enforced by the HRT library Nilsson et al. (2004), giving a precision of up to $1.9 \mu \mathrm{s}$. Sample-and-hold interpolation was used to find synchronised values for the electrodes and glove sensors. All data were collected in batches, each one labelled with a corresponding subject and grasp index.

As the setup did not include any way to indicate precisely when the carrying phase would happen (i.e. an instrumented object or a pressure sensor), a manual procedure was enforced offline to isolate it for each sequence. During the procedure, the value of the glove index finger sensor and the sum-of-squares of the sEMG electrodes were visualised; the experimenter would then identify and note the intervals corresponding to the carrying phase.

An example sequence (subject 1 doing a flat grasp) is shown in Fig. 4. Correlation is apparent between the two signals; the high-valued periods denote the carrying phase, when muscle activity was maximum and the index finger would be flexed over the object, as opposed to the resting periods, characterised by low muscle activity and the index standing in the resting position (low values). It must be noted that in other cases the correlation is not apparent and that is why this process had to be manually enforced. For instance, in

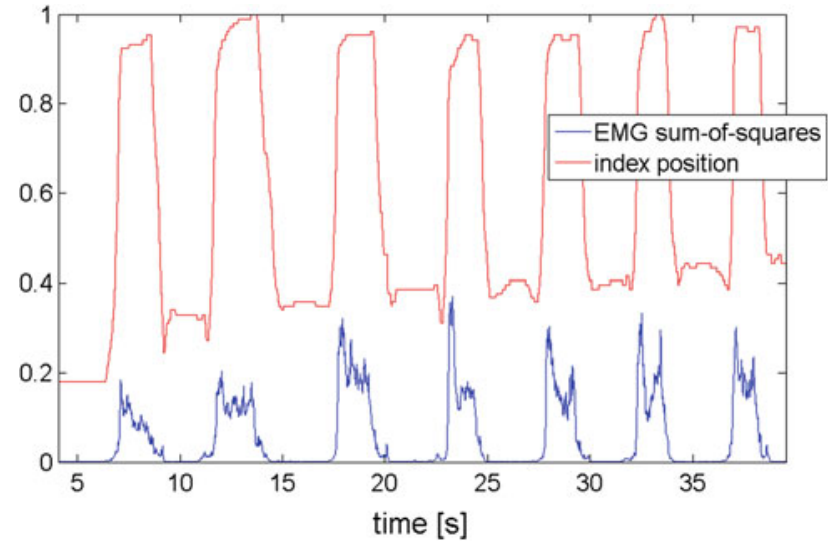

Fig. 4 Typical 'good' grasping sequence (subject 1, flat grasp); correlation is apparent between the sEMG power and the index finger position

the large-power grasp case, the index finger would assume a lower value during lifting than in the resting phase. Moreover, some of the lifting periods were not characterised by enough muscle activity or by the expected kinematic postures due to local failure of the sensors. The experimenter excluded these sequences from the analysis. As well, subject 4 exhibited little or no measured muscle activity so these data were removed from the analysis too. This was later on determined to be due to inaccurate sensor placement at the beginning of the procedure.

At the end $97 \%$ of the original data, that is 487 lifting intervals out of the expected 500 were identified: 5 subjects repeating each of the 5 grasps for 20 times. Data were then normalised by subtracting the mean values and dividing by the standard deviations, dimension-wise, per-subject, to remove the intra-subject differences due to the hand size and the level of muscle fitness.

Finally, each sequence was averaged out dimension-wise. These average values were assumed as representatives of stable grasps enforced during each carrying phase. This operation resulted then in 487 new samples, each one denoting a sequence labelled by a subject and grasp index. For each sample, two sets of features were obtained: the 18dimensional kinematic features obtained from the glove, and the 10-dimensional $s E M G$ features obtained from the sEMG electrodes. Additionally, sometimes only the proximal or the distal electrodes have been considered, and both sets are 5-dimensional.

Two sets of labels were obtained, one according to the subjects $(1,2,3,5,6)$ and one according to the grasps (flat grasp, pinch grip, tripodal grip, small power grasp and large power grasp, numbered as $1,2,3,4,5)$. In mathematical terms, we have built four sets of samples $X_{\text {glove }}, X_{\mathrm{EMG}}, X_{\text {dist }}$ and $X_{\text {prox }}$, each one containing 487 elements, denoted as $X=\left\{\left(\mathbf{x}_{i}\right)\right\}_{i=1}^{n}$, where $n=487$ and $\mathbf{x}_{i} \in \mathbb{R}^{d}$, where $d$ is 18 for $X_{\text {glove }}, 10$ 
for $X_{\mathrm{EMG}}$ and 5 for $X_{\text {dist }}$ and $X_{\text {prox }}$. Two sets of labels are then built, $Y_{\text {subj }}$ and $Y_{\text {grasp }}$ where $Y=\left\{\left(y_{i}\right)\right\}_{i=1}^{n}$ and $y_{i} \in[1,2,3,5,6]$ for $Y_{\text {subj }}$ and $y_{i} \in[1,2,3,4,5]$ for $Y_{\text {grasp. }}$.

\section{Experimental results}

\subsection{Kinematic synergies and muscle synergies}

Principal component analysis (PCA), a very basic dimensionality reduction technique (see, e.g. Duda et al. (2001)), was first applied to the dataset to check that a small number of linear combinations of kinematic and/or sEMG features would account for a reasonable amount of variability in the data set $^{1}$. In a nutshell, PCA works by first sphering the sample matrix $X$ (i.e. subtracting from it the average sample values dimension-wise and then slashing it by the standard deviations), then evaluating its covariance matrix $\Sigma=\frac{1}{n-1} X^{T} X$. $\Sigma$ is then decomposed according to the single-value decomposition,

$$
\Sigma=U \bar{\Sigma} V^{T}
$$

where $\bar{\Sigma}$ is diagonal and contains the eigenvalues of $\Sigma$. The columns of $U$ are rearranged to match the magnitude of the eigenvalues in decreasing order so that each of these columns, when applied back to $X$, produces a linear combination of the dimensions of $X$ having a decreasing degree of signal variance. Denoting by $U_{k}$ the $k \times n$ submatrix of $U$ obtained by selecting only the first $k$ columns of $U$,

$X^{*}=U_{k} X$

is a $k$-dimensional projection of $X$ onto a space preserving a certain, maximal fraction of the signal variance in $X$ (where $k<d$-a superscript asterisk denotes the projected data onto $k$ dimensions). By looking at the cumulative sum of the eigenvalues of $\Sigma$, we can detect how much signal variance is lost as $k$ is increased until $k=d$. Each of the $k$ dimensions is called a Principal component of $X$. In our case, principal components of sEMG or glove values denote the (linear) synergistic sEMG/kinematic activity. (From now on then, principal components will be denoted as kinematic or sEMG synergies.)

Consider Fig. 5, showing the percentage of data variance as more and more synergies are considered. On the left panel, PCA is applied to the whole dataset altogether, irrespective of subjects and grasps. In the case of kinematic features (glove sensors, $X_{\text {glove }}$ ), three synergies account for $74.62 \%$ of the total signal variance; five of them account for $85.52 \%$. In the case of sEMG features $\left(X_{\mathrm{EMG}}, X_{\text {dist }}, X_{\text {prox }}\right)$, the figures for

\footnotetext{
${ }^{1}$ Recall that from now on we will be using the dataset obtained by averaging out the sensor values over the carrying phases identified during the preprocessing phase.
}

three synergies rise to 92.63 and $91.11 \%$ (in turn, $X_{\text {prox }}$ and $\left.X_{\text {dist }}\right)$ and $83.62 \%\left(X_{\mathrm{EMG}}\right)$.

This clearly proves that kinematic synergies are present; our figures are in agreement with previous work, given the simplicity of the tasks at hand (e.g. Santello et al. (1998)). The grasps considered can be captured (at $75 \%$ variance) by three linear combinations of $X_{\text {glove }}$, meaning that most of the grasps share the same three characteristic 'eigengrasps'.

A more interesting result is that very strong $s E M G$ synergies are found as well; that is, that muscles, as represented by their sEMG values, act in a mostly coordinated fashion, exerting the same forces over and over again. Three linear combinations of the 10 electrodes considered account for slightly less than $84 \%$ of the whole signal variance.

Consider now the right panel of the same Figure. This time both kinematic and sEMG data have been grouped per-subject (markers and error bars denote average variance percentage values plus/minus one standard error of the mean). In this case, the 'compression' obtained by three synergies is even more evident, being in turn $87.37 \pm 1.5 \%$, $96.36 \pm 0.72 \%, 95.13 \pm 1.23 \%$ and $91.46 \pm 1.41 \%$ for $X_{\text {glove }}, X_{\text {prox }}, X_{\text {dist }}, X_{\text {EMG }}$. This result overall means that both kinematic and muscle synergies exist, and that they are even stronger at the single-subject level.

\subsection{Common synergy features}

We now turn to a more qualitative analysis of the synergies found in the previous Subsection. From now on, we will consider 3 synergies only, that is, $k=3$-this has the great advantage that data can be visualised, and it involves an acceptable loss of information as previously stated. We first focus on a reduced version of the problem, namely we consider a subset of three grasps: pinch grip, small power grasp and large power grasp (second, fourth and fifth panels from the left of Fig. 3). These grasps are very different from one another from a kinematic point of view; this is reflected in their distance in standard grasp taxonomies (examples can be found in (Cutkosky 1989; Kang and Ikeuchi 1993)).

Consider first Fig. 6, depicting the grasps in 3 dimensions (first, second and third synergy) in the kinematic (left, plotting data from $X_{\text {glove }}^{*}$ ) and sEMG (right, plotting data from $X_{\mathrm{EMG}}^{*}$ ) spaces. It is apparent that the grasps are well clustered to the point that a linear classifier (i.e. a plane in 3D) could separate them perfectly from one another in kinematic space, and almost perfectly in muscle space. As opposed to this, consider now Fig. 7 which depicts the same data, but associating a colour to each subject (rather than to each grasp): separability is much less clear. Visual comparison with Fig. 6 indicates that each subject participates in each of the 3 clusters associated with the grasps.

In other words, grasps can be distinguished, but subjects can not. All subjects roughly do the same things when, e.g. 


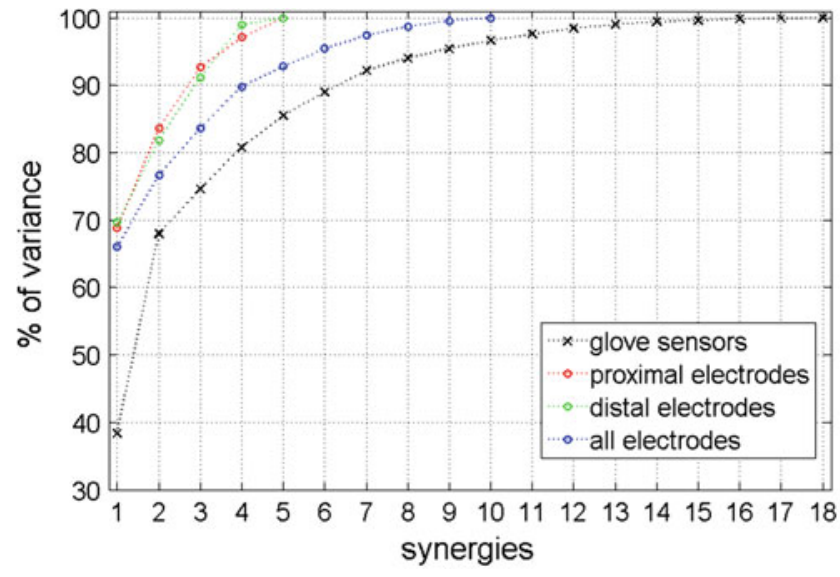

Fig. 5 Principal component analysis of kinematic $\left(X_{\text {glove }}\right)$ and sEMG features $\left(X_{\mathrm{EMG}}, X_{\mathrm{dist}}, X_{\text {prox }}\right)$ as $k$ is increased; the plots show the normalised cumulative sum of the PCA eigenvalues. (left) All samples

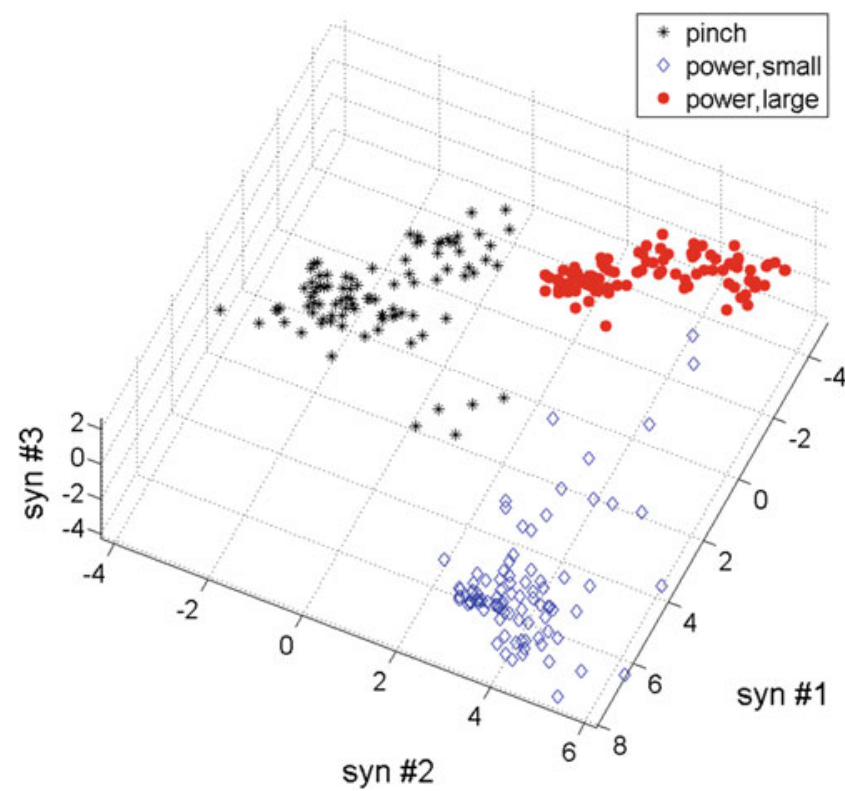

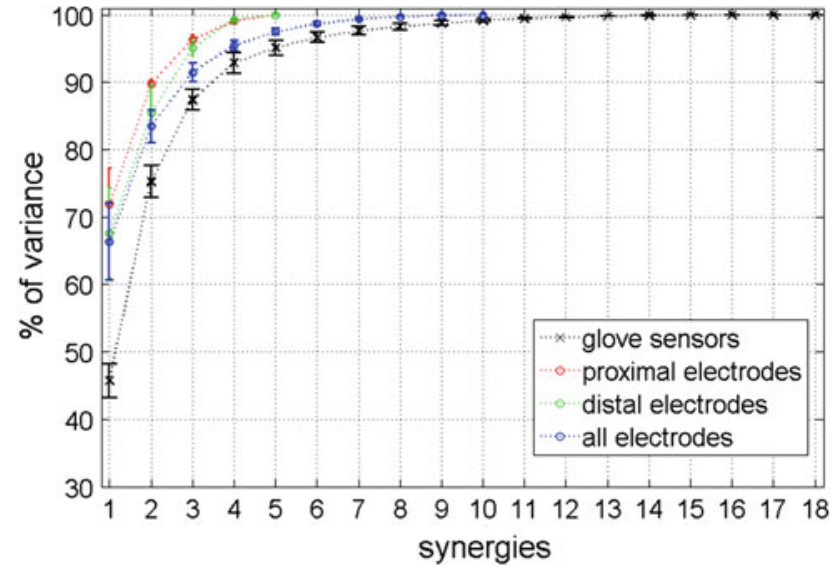

considered altogether; (right) grouped by subject, markers and error bars denoting average values plus/minus one standard error of the mean

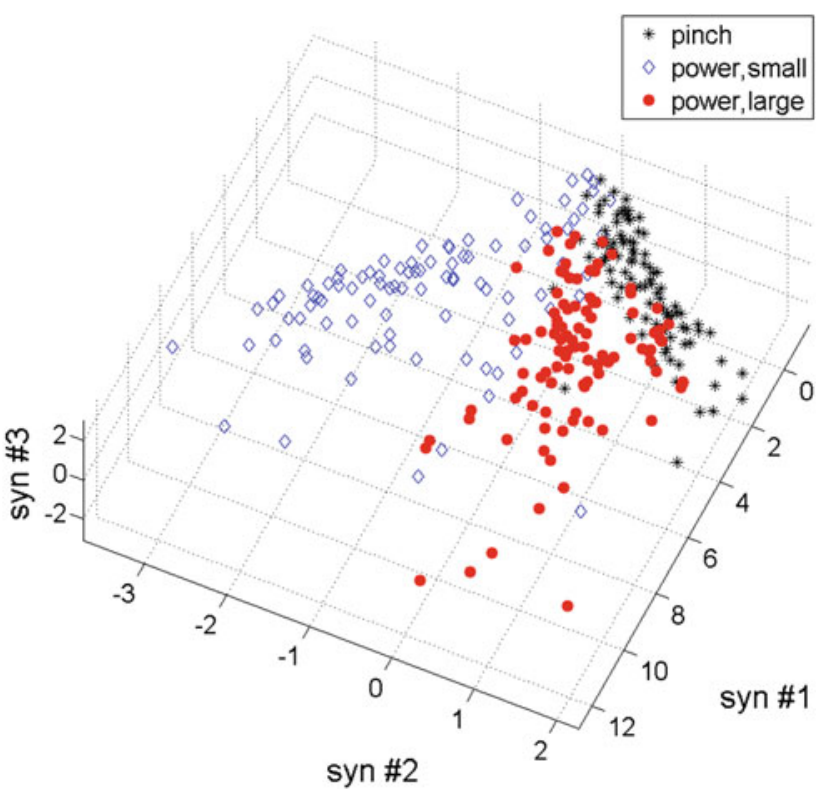

Fig. $63 \mathrm{D}$ visualisation of 3 of the grasps as performed by all subjects; colours denote grasps. Kinematic synergies (plotting samples in $X_{\text {glove }}^{*}$, left); muscle synergies, using all electrodes altogether $\left(X_{\mathrm{EMG}}^{*}\right.$, right)

pinch gripping, both kinematically (and this is no surprise) and as far as muscle activity is concerned. In order to numerically verify this statement, we now turn to the fully fledged problem (five grasps, five subjects, 487 samples). For each of the above-described settings (each setting is mathematically represented by a sample set and a label set), we ran a multiclass linear classifier and considered the balanced error rate (BER) as a measure of separability of grasps and subjects. The BER is defined as

$\mathrm{BER}=\frac{1}{5} \sum_{j=1}^{5} \frac{c_{j}}{l_{j}}$ where $c_{j}$ denotes the correctly predicted labels for class $j$ and $l_{j}$ is the total number of labels for class $j$.

Linear classification is a statistical technique which can be used, at a very basic level such as this, to check how separated $N$ classes of objects are (see, e.g., the classic Duda et al. (2001) again); in particular, for a sample/label set pair $(X, Y)$, a linear classifier will here find a set of 3D planes such that all samples in $X$ associated to a label $y_{i} \in Y$ will be on one side of the plane, whereas samples belonging to all other categories will be on the other side. As a linear classifier, we chose to use a support vector machine (SVM) by linear kernel. SVMs Boser et al. (1992); Vapnik (1998) are a machine learning method which will find the separating 

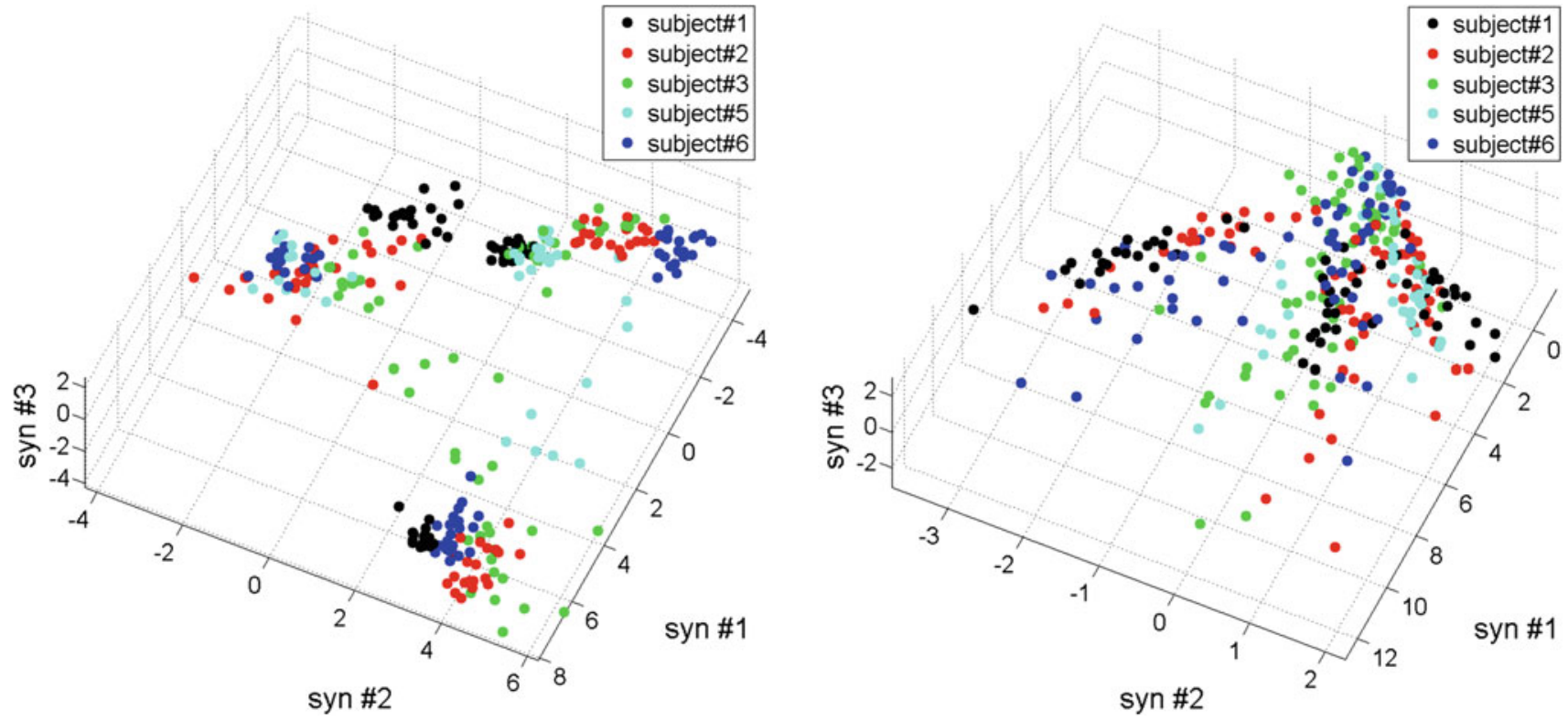

Fig. 7 3D visualisation of 3 of the grasps as performed by all subjects; colours denote subjects. Kinematic synergies ( $X_{\text {glove }}^{*}$, left); muscle synergies $\left(X_{\mathrm{EMG}}^{*}\right.$, right)

(hyper)plane between two sets of labelled sample, such that the margin between the categories is maximised. By margin here it is meant, twice the distance between the separating plane and the closest sample in either category. The plane thus found enjoys maximum robustness against noise in the sampling procedure (Burges 1998); in this sense, it is the optimal separating plane. In mathematical terms, given samples and labels $\left\{\left(\mathbf{x}_{i}, y_{i}\right)\right\} \in(X, Y)$, the separating plane is

$f(\mathbf{x})=\mathbf{w} \cdot \mathbf{x}+b=\sum_{i=1}^{n} \alpha_{i} y_{i}\left(\mathbf{x} \cdot \mathbf{x}_{i}\right)+b$

where $\alpha_{i}, \mathbf{w} \in \mathbb{R}^{d}$ and $b \in \mathbb{R}$. The $\alpha_{i}$ s and $b$ are found by minimising a regularised loss function

$L(\mathbf{w}, \boldsymbol{\alpha}, b)=\left\{\frac{1}{2}\|\mathbf{w}\|^{2}+C \cdot L(\mathbf{w}, b, Y)\right\}$

with $C \geq 0 \in \mathbb{R}$.

Since our dataset $S$ consists of a relatively small number of samples (478) we employed twofold cross-validation and grid search to find the optimal SVM $C$ hyperparameter; that means that a randomly chosen half-sized subset of $S$ was employed for training and the remaining half was used to test. The procedure was run for 50 times, each time with a different random choice of the training/testing sets and then displaying the means and standard deviations of the errors so obtained. Table 1 shows the results.

As is clear from the Table, trying to tell subjects from one another is pointless, as all error rates approach the chance level of $80 \%$ (recall that there are 5 subjects). As opposed to that, grasps can be distinguished quite well; in particular, kinematic synergies represent an almost perfect set of discriminating features (see Fig. 6 (left) again). Also, using the $10 \mathrm{sEMG}$ electrodes altogether an error rate of $6.39 \% \pm 1.8, \%$ is achieved.

The same trend is visible when considering Table 2, in which all grasps are considered. Results here are uniformly worse, as one would expect since flat grasp, pinch grip and tripodal grip are quite similar to one another. Still the trend of Table 1 is visible, subject discrimination being uniformly worse than grasp discrimination. The sEMG features ( $X_{\mathrm{EMG}}, Y_{\text {grasp }}$ ) achieve a BER of $33.49 \pm 2.50 \%$, which is still significantly better than chance level.

Notice that correct classification of sEMG patterns is out of the scope of this paper-that has already been done with greater success, e.g. by SVMs with Gaussian kernels. The interest of the result presented above lies rather in the statistically significant separability of one or more set(s) of samples. In this case, an error rate below chance level is already meaningful. Linear separability here points at the common pattern underlying a certain class; for instance, the fact that a plane can separate the large power grasp from the pinch grip irrespective of the subjects means that an easy procedure can be found to tell which grasp is being enacted (for instance, evaluating the sample distance from the plane itself).

\subsection{Inter-synergy distances}

The above results clearly establish that strong synergies exist both at the kinematic and muscular levels during grasping, 
Table 1 Balanced error rates obtained while applying a linear classifier to one of the $(X, Y)$ sample/label sets shown in Figs. 6 and 7 . Considering three grasps: pinch grip, small power grasp and large power grasp

\begin{tabular}{lllll}
\hline & Kinematic $\left(X_{\text {glove }}, \%\right)$ & sEMG, elbow $\left(X_{\text {prox }}, \%\right)$ & sEMG, forearm $\left(X_{\text {dist }}, \%\right)$ & sEMG, all $\left(X_{\text {EMG }}, \%\right)$ \\
\hline Grasps $\left(Y_{\text {grasp }}\right)$ & $0.38 \pm 0.38$ & $13.87 \pm 2.29$ & $17.13 \pm 2.22$ & $6.39 \pm 1.87$ \\
Subjects $\left(Y_{\text {subj }}\right)$ & $60.13 \pm 6.29$ & $57.15 \pm 5.43$ & $74.21 \pm 3.71$ & $67.80 \pm 5.92$ \\
\hline
\end{tabular}

Table 2 Balanced error rates obtained while applying a linear classifier to one of the $(X, Y)$ sample/label sets shown in Figs. 6 and 7 . Considering all grasps

\begin{tabular}{lllll}
\hline & Kinematic $\left(X_{\text {glove }}, \%\right)$ & sEMG, elbow $\left(X_{\text {prox }}, \%\right)$ & sEMG, forearm $\left(X_{\text {dist }}, \%\right)$ & sEMG, all $\left(X_{\text {EMG }}, \%\right)$ \\
\hline Grasps $\left(Y_{\text {grasp }}\right)$ & $18.54 \pm 2.01$ & $35.46 \pm 3.05$ & $46.28 \pm 3.30$ & $33.49 \pm 2.50$ \\
Subjects $\left(Y_{\text {subj }}\right)$ & $77.06 \pm 2.79$ & $59.45 \pm 3.57$ & $71.99 \pm 2.80$ & $64.25 \pm 4.35$ \\
\hline
\end{tabular}

and that they can be effectively used to characterise each grasp across subjects. A further interesting question is that of checking whether the grasps look similar in the two spaces or not. It is expected that similarities should exist in both spaces, but that they should be different according to the space.

In order to investigate this issue, we consider the mean values of each group of samples representing a grasp, both in the kinematic and sEMG space; this corresponds to considering the centres-of-mass of the clusters of Fig. 6 (considering all the five grasps, rather than just three). We then evaluate the pairwise Euclidean distance between these centres, obtaining two inter-grasp distance matrices, visible in Fig. 8. In each matrix $D$, element $D_{i j}$ represents the Euclidean distance between grasp $i$ and grasp $j$ in the related space. Distances are normalised between 0 and 1. (The matrices are obviously symmetric.)

As is apparent from the matrices, the inter-grasp distances are not quite similar in the two spaces. For instance, in the kinematic space the small and large power grasps have 0.39 distance, whereas in the sEMG space this figure is 1 , that is, they are the two most different grasps. This indicates that the two grasp postures are very different (completely open versus completely closed), but the patterns of muscle activation are similar. A striking example of the opposite phenomenon is the distance between pinch grip and large power grasp: kinematically there is little difference (distance 0.35, all fingers wrapped around something), but a completely different muscle configuration (distance 1 ) is employed. Finally, notice that pinch grip and tripodal grip are essentially identical from a kinematic point of view (distance 0.01), but rather different in sEMG space (distance 0.08). Again, this is sensible, since activating the middle finger requires additional muscle power, whereas the two grips look very similar as far as the hand posture is concerned.

\subsection{Anatomical relevance of synergies}

Finally, we restrict our attention to the sEMG synergies (that is, to the columns of $U_{k}$ ) and try to understand what they mean from a muscular / anatomical point of view. As stated above, the placement of electrodes is irrespective of the anatomy, but it was kept carefully uniform across all subjects; i.e. the ventral electrodes were always placed over the ventral muscles (flexors), the dorsal electrodes over the extensors and the distal electrodes were always placed farther away from the elbow with respect to the proximals. In general, muscle synergies are linear combinations of electrode values which maximise the signal variance; they reflect the grouped activation of the forearm muscles. Therefore, inspecting these combinations should reveal in which ways groups of muscles are activated during grasping.

In order to investigate this issue, the first three columns of $U_{k}$ are projected back onto the sets of sEMG electrodes, thus retrieving the main modes of electrode activation, as they are spatially distributed over the forearm of the subjects. Figure 9 shows the obtained activations as a polar graph, for each synergy. Values are normalised between -1 and 1.

In order to facilitate the inspection of the graphs, the distal and proximal electrode activations are represented with the same colour in two different shades: normal for the proximal electrodes and light for the distal ones. For instance, the activation of proximal electrodes in synergy \#1 is red, whereas that of distal electrodes is light red. Moreover, dorsal electrodes are shown on the right-hand side of the graphs whereas ventral electrodes appear on the left, coherently with the steady forearm posture assumed by the subjects during the carrying phase; each polar graph can be superimposed to a forearm going through the page, ventral side on the left. See again Figs. 1 (right panel) and 2, and the movie 'grasping.avi'. 

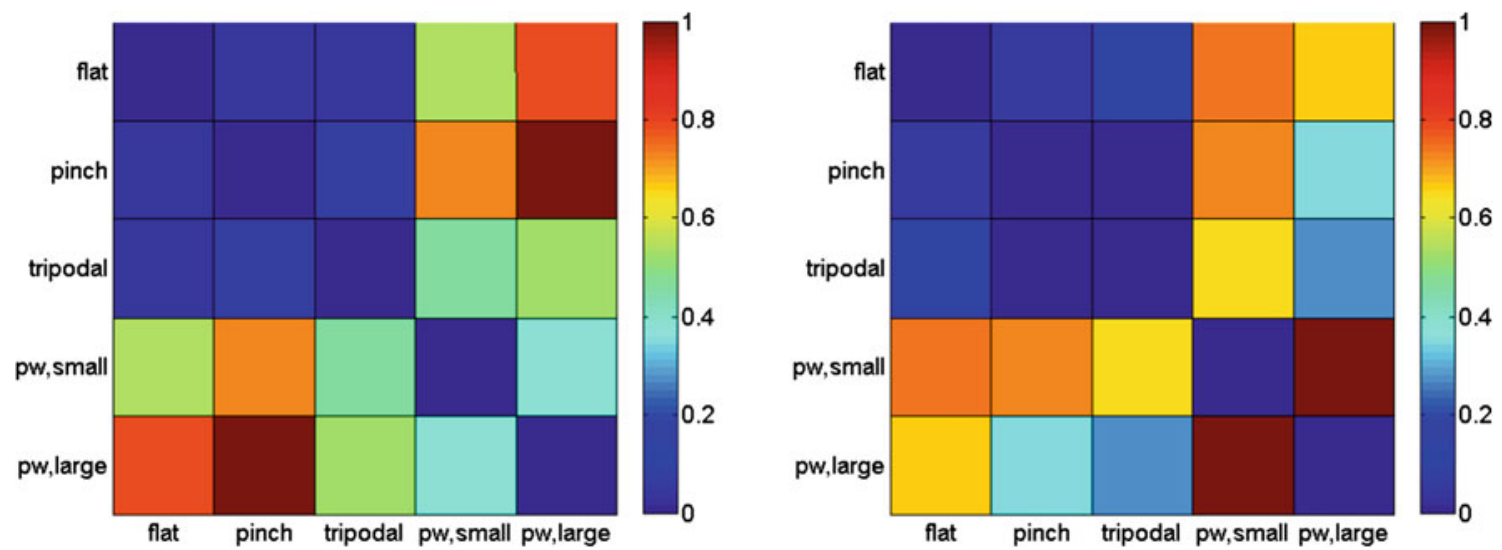

Fig. 8 Inter-grasp distance matrices. Grasps projected onto 3 muscle synergies (left); onto 3 kinematic synergies (right). Distances are normalised between 0 and 1

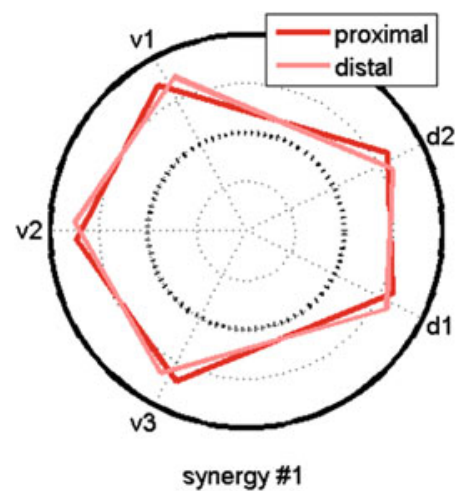

Fig. 9 The first three muscle synergies (first three columns of $U_{k}$ ) represented on an electrode-activation axis. The graphs show the dorsal electrodes ( $\mathrm{d} 1$ and $\mathrm{d} 2$ ) on the right-hand side, and the ventral electrodes (v1, v2 and v3) on the left. Proximal electrodes are represented in full-

The first remark is that proximal and distal electrodes are always activated coherently; for example, the dorsal 2 position (d2) shows high values in synergy \#1 and \#2 and low values in synergy \#3, for both the proximal and distal electrodes; the dorsal 1 is low in synergies \#2 and \#3 and high in synergy \#1; and so on. The shapes of the light- and darkcoloured lines match uniformly. This is expected, since in the forearm, muscles stretch along the forearm axis; therefore, similar, coherent levels of activation are expected if we gather the activation potential in different spots along the axis itself, but without rotating the electrodes position. This also validates the constraint that the forearm must not be pronated/supinated during the experiment.

As far as synergies are concerned, synergy \#1 represents a uniform activation of all muscles, flexors (ventral side) and extensors (dorsal side). This is caused by the coactivation of the extensor and flexor muscles in every finger flexion and extension, and reflects the complexity of the complex underlying hand biomechanics, i.e. the connection between the tendons themselves as well as between the tendons and the joints. Tendons usually have multiple insertion points in the finger, and the stable movement of a finger involves the coactivation of many muscles to prevent instability of the same as well as of the other fingers.

On the other hand, synergies \#2 and \#3 take into account more prominent activations of, in turn, the upper dorsal (d2) and the upper ventral (v1, v2) sides. This is correlated to the selective activation of the extensor muscles during pinch grip (to refrain from clutching an object with all fingers) and other similar grips (for instance, the tripodal).

This is confirmed by Fig. 10, in which each of the five grasps considered is projected onto the three muscle synergies of Fig. 9-in other words, the grasp components along the three synergies are visualised. As one can see, synergy \#1 is maximally active in the power grasps, much less in the tripodal and minimally in the pinch and tripodal grip. 


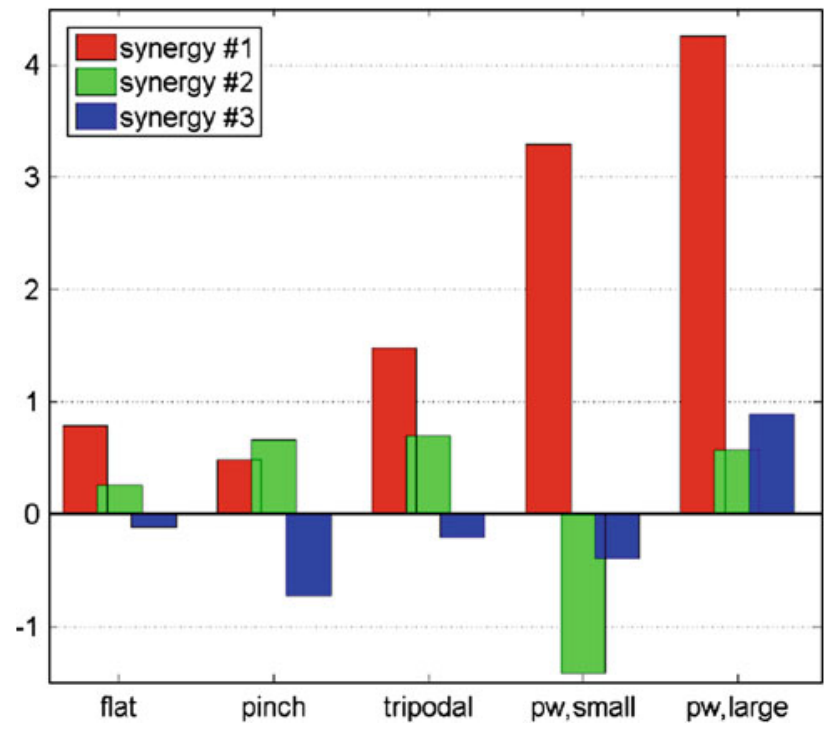

Fig. 10 The five grasps, expressed in terms of the three muscle synergies visible in Fig. 9

We interpret this as reflecting the uniform activation of the muscles when a cylindrical or spherical grasp is required. In these cases, there is no selective activation on one side or the other. Remarkably, the tripodal grip shows a much higher usage of this synergy with respect to the pinch grip. As opposed to that, synergies \#2 and \#3 are activated in the same order of magnitude of synergy \#1 only in the cases of the flat and pinch grips, where a really selective activation of two or three fingers (and therefore of different parts of the flexors/extensors) is required.

\section{Conclusions and discussion}

\subsection{Evidence of muscle synergies}

The concept of synergies has since long been established in the kinematic description of the human hand. Indeed, when taking a large number of everyday grasps into account, most movements of the fingers of the hand can be described with a very limited number of principal components (i.e. 3-5 Santello et al. (1998), considerably less than the number of degrees of freedom of all fingers combined, i.e. 25 Stillfried and van der Smagt (2010)). Complementary to that, this work tries to answer the following, so-far unanswered question: are analogous synergies also present at the level of the exerted grasp forces and, consequently, of the activation of the forearm muscles?

Our results confirm that, at least in this experiment, and using surface electromyography, this is the case. Muscle activation data, i.e. the sEMG signal gathered from 5 human subjects during a grasping task, can be represented using as few as 3 principal sEMG components (muscle synergies) while retaining $85-90 \%$ of the signal variance. Moreover, the signal samples can be effectively linearly separated when clustered on a per-grasping basis, but not on a persubject basis. From this, we conclude that as few as 3 sEMG synergies are sufficient to characterise one among 5 grasps, with a precision which obviously depends on the (muscular) similarity among grasps, but that anyway largely surpasses the corresponding precision when trying to discriminate subjects.

The fact that we employ such a simple classification system as a linear discriminant (Support Vector Machine with linear kernel) proves that the separation is not only above chance level but also evident. Analogously to what happens for kinematic synergies, there are muscle synergies in humans; and when humans engage in a grasping task, they are characteristic of grasp types, but not of subjects. In one word, they are largely invariant across (our) subjects.

\subsection{Anatomical relevance of muscle synergies}

Kinematic synergies are evidently related to the shape of the objects which are being grasped, meaning that their combination leads to the same hand shapes all over. An analogous, but different, story emerges from the qualitative analysis of muscle synergies. In our experiment, the sEMG electrodes were placed at anatomically similar locations in all subjects, without targeting single muscles but rather groups of them, namely the flexors (on the ventral side of the forearm) and the extensors (on the dorsal side). By back-projecting the 3 sEMG principal components on the electrode layout (consider Fig. 9 again), we discovered that each synergy corresponds to a qualitatively very different synergistic muscle activation: uniform activation, activation of the dorsal muscles near the radius and activation of the flexors near the radius.

A description of the grasps according to muscle synergies confirms that each grasp employs each synergy to very different degrees. (This analysis reminds of that found for kinematic synergies in, e.g. (Santello et al. 1998, 2002; Bicchi et al. 2011).) From this, we conclude that muscle synergies are physiologically plausible representations of the grasps themselves, or rather, of the muscle activations underlying them. As an aside note, it would be interesting to validate this grasp/activation correspondence by comparing it to a pre-existing analogous grasp taxonomy/hierarchy. We could not find anything as such in the literature, nor we could use to this aim any well-known taxonomy such as, e.g. Cutkosky's Cutkosky (1989) or Kang's Kang and Ikeuchi (1993), which assume task-oriented and purely kinematic points of view. 


\subsection{Kinematic and muscle synergies}

Inter-pattern distances (consider Fig. 8 again) show that force-similar grasps are close to each other in the 3-principalcomponent sEMG space, analogously to what happens in the kinematic (Cyberglove data) space, and that the sEMG and kinematic distance matrices are very different from each other, as it is intuitively expected. Grasps which require similar muscle activations do not necessarily match pairs of grasps which correspond to similar finger positions.

This sheds some light on the standard approach to grasp planning, in which mostly a purely kinematic stance is assumed. During the reaching/pre-shaping phase, it is probably best to work with the hand configuration (finger positions) to match the shape of the object to be grasped; but, to then enforce grasp stability it might be useful to also consider the required force configuration at the fingertips. Such a force configuration might effectively be planned in advance using the sEMG signal of a human subject, for instance, during teleoperation, mapped onto the dynamics of the robotic gripper. Indeed, in Brochier et al. (2004) it was shown that different EMG signals related to different objects differed not only during grip but also during the reaching movement towards the object.

\subsection{Applications}

This result is still mainly at the level of basic research; in order for it to be applicable, the issues of stability and generality of human muscle synergies must be first investigated. Firstly, the recurrence of (at least) comparable synergies across sessions of data gathering must be assessed. The sEMG of a single subject is notoriously prone to wide changes as the subject sweats, as electrode positions vary when they are donned and doffed, and as muscle fatigue appears. The situation is even worse when comparing the signal across subjects, given the natural variability of human anatomy. Finally, one of the main target applications of this work is about amputees, and every amputation is in general different from all others, making it quite unrealistic that previous knowledge extracted from a pool of subjects (either amputated or intact) will generalise to new amputees.

Actually, the use of surface EMG is, from this point of view, a hindrance; the problem of knowledge transfer across subjects has already been investigated in this field (Castellini et al. 2009) with rather negative results, at least using a naive application of previously trained models on new subjects. A more sophisticated technique has been used with better success in Orabona et al. (2009); Tommasi et al. (2012), but in that case the subject pool was remarkably larger. An even larger pool of subjects is envisioned to be used in the Ninapro project (Atzori et al. 2012).
Of course, one slightly less comfortable, but feasible, scenario is that of training the classifier for every subject before going into action. In this case, the situation seems much better even for amputees: residual, stable muscle activity of excellent quality has recently been found in long-term amputees (Sebelius et al. 2005; Castellini et al. 2009; Tenore et al. 2009; Cipriani et al. 2011).

Having said that, the use of sEMG synergies finds it main application in force- and impedance-based control of mechanical and prosthetic hands. By considering the sEMG synergies when detecting muscular activity and controlling the prosthetic hand correspondingly, a more robust approach to sEMG-based hand prosthesis control may be obtained; in particular, even mechanical hands gifted with a high number of degrees of freedom can be controlled with a reduced set of commands (Wimböck et al. 2011).

Acknowledgments This work is partially supported by the European FP7-Project THE Hand Embodied (FP7-IST-248257) and by the Swiss National Science Foundation Sinergia project \#132700, Ninapro (NonInvasive Adaptive Prosthetics). The authors would also like to thank Mr. Johann Buchner of the DLR for building some of the electronics involved in the setup. Legal compliance the authors declare that the experiment described in this paper complies with the current relevant German laws.

\section{References}

Arbib M, Metta G, van der Smagt P (2008) Neurorobotics: from vision to action, Chap. 62. Springer, Berlin, pp 1453-1480

Atzori M, Gijsberts A, Heynen S, Mittaz-Hager AG, Deriaz O, van der Smagt P, Castellini C, Caputo B, Müller H (2012) Building the NINAPRO database: a resource for the biorobotics community. In: Proceedings of BioRob-IEEE international conference on biomedical robotics and biomechatronics, pp 1258-1265

Bernstein N (1967) The coordination and regulation of movements. Pergamon Press, Oxford

Bicchi A, Gabiccini M, Santello M (2011) Modelling natural and artificial hands with synergies. Philos Trans R Soc London. Ser B, Biol Sci 366(1581):3153-3161. doi:10.1098/rstb.2011.0152

Boser BE, Guyon IM, Vapnik VN (1992) A training algorithm for optimal margin classifiers. In: Haussler D (ed) Proceedings of the 5th annual ACM workshop on computational learning theory, ACM press, pp 144-152

Brochier T, Spinks RL, Umiltà MA, Lemon RN (2004) Patterns of muscle activity underlying object-specific grasp by the macaque monkey. J Neurophysiol 92(3):1770-1782

Burges CJC (1998) A tutorial on support vector machines for pattern recognition. Knowl Discov Data Min 2(2):955-974

Castellini C, Fiorilla AE, Sandini G (2009) Multi-subject/daily-life activity EMG-based control of mechanical hands. J Neuroeng Rehabil 6(41). doi:10.1186/1743-0003-6-41.

Castellini C, Gruppioni E, Davalli A, Sandini G (2009) Fine detection of grasp force and posture by amputees via surface electromyography. JPhysiol 103(3-5):255-262. doi:10.1016/j.jphysparis.2009.08.008

Castellini C, van der Smagt P (2009) Surface EMG in advanced hand prosthetics. Biol Cybern 100(1):35-47. doi:10.1007/ s00422-008-0278-1

Castellini C, van der Smagt P (2011) Preliminary evidence of dynamic muscular synergies in human grasping. In: Proceedings of 
ICAR - international conference on, advanced robotics, pp 28-33 doi:10.1109/ICAR.2011.6088612

Cipriani C, Antfolk C, Controzzi M, Lundborg G, Rosén B, Carrozza M, Sebelius F (2011) Online myoelectric control of a dexterous hand prosthesis by transradial amputees. IEEE Trans Neural Syst Rehabi Eng 19(3):260-270. doi:10.1109/TNSRE.2011.2108667

Cutkosky M (1989) On grasp choice, grasp models, and the design of hands for manufacturing tasks. IEEE Transa Robot Autom 5(3): 269-279

De Luca CJ (1997) The use of surface electromyography in biomechanics. J Appl Biomech 13(2):135-163

De Luca CJ (2002) Surface electromyography: detection and recording. Copyright 2002 by DelSys, Inc.

Duda RO, Hart PE, Stork DG (2001) Pattern classification, 2 edn. Wiley, New York

Grebenstein M, van der Smagt P (2008) Antagonism for a highly anthropomorphic hand-arm system. Adv Robot 22(1):39-55. doi:10.1163/ $156855308 X 291836$

Grinyagin IV, Biryukova EV, Maier MA (2005) Kinematic and dynamic synergies of human precision-grip movements. J Neurophysiol 94(4):2284-2294

Nawab HS, Wotiz RP, De Luca CJ (2008) Decomposition of indwelling EMG signals. J Appl Physiol 105:700-710

Holdefer RN, Miller LE (2002) Primary motor cortical neurons encode functional muscle synergies. Exp Brain Res 146(2):233-243

Kang SB, Ikeuchi K (1993) A grasp abstraction hierarchy for recognition of grasping tasks from observation. Proc. IEEE/RSJ Int'l Conf. on Intelligent Robots and Systems, Yokohama

Lang CE, Schieber MH (2004) Human finger independence: limitations due to passive mechanical coupling versus active neuromuscular control. J Neurophysiol 92(5):2802-2810

Mussa-Ivaldi FA, Giszter SF, Bizzi E (1994) Linear combinations of primitives in vertebrate motor control. Proc Natl Acad Sci USA 91(16):7534-7538

Nilsson J (2004) Implementing a continuously updating, highresolution time provider for windows. The MSDN Magazine

Orabona F, Castellini C, Caputo B, Fiorilla E, Sandini G (2009) Model adaptation with least-squares SVM for hand prosthetics. In: Proceedings of ICRA-International Conference on Robotics and Automation, pp 2897-2903 doi:10.1109/ROBOT.2009.5152247.
Overduin SA, d'Avella A, Roh J, Bizzi E (2008) Modulation of muscle synergy recruitment in primate grasping. J Neurosci 28(4):880-892

Santello M, Flanders M, Soechting JF (1998) Postural synergies for tool use. Neuroscience 17:10105-10115

Santello M, Flanders M, Soechting JF (2002) Patterns of hand motion during grasping and the influence of sensory guidance. Neuroscience 22(4):1426-1435

Sebelius FCP, Rosén BN, Lundborg GN (2005) Refined myoelectric control in below-elbow amputees using artificial neural networks and a data glove. J Hand Surg 30(4):780-789

Stillfried G, van der Smagt P (2010) Movement model of a human hand based on magnetic resonance imaging (MRI). International Conference on Applied Bionics and Biomechanics (ICABB), Venice

Takei T, Seki K (2010) Spinal interneurons facilitate coactivation of hand muscles during a precision grip task in monkeys. J Neurosci 30(50):17041-17050

Tenore FV, Ramos A, Fahmy A, Acharya S, Etienne-Cummings R, Thakor NV (2009) Decoding of individuated finger movements using surface electromyography. IEEE Trans Biomed Eng 56(5): $1427-1434$

Tommasi T, Orabona F, Castellini C, Caputo B (2012) Improving control of dexterous hand prostheses using adaptive learning. IEEE Transactions on Robotics. doi:10.1109/TRO.2012.2226386

Tsuji H, Ichinobe H, Ito K, Nagamachi M (1993) Discrimination of forearm motions from emg signals by error back propagation typed neural network using entropy. IEEE Trans Soc Instrum Control Eng 29(10):1213-1220

Vapnik VN (1998) Stat Learn Theory. Wiley, New York

Vogel J, Castellini C, van der Smagt P (2011) EMG-based teleoperation and manipulation with the DLR LWR-III. In: Proceedings of IROS -international conference on intelligent robots and systems, pp 672-678. doi:10.1109/IROS.2011.6048345

Wimböck T, Jahn B, Hirzinger G (2011) Synergy level impedance control for multifingered hands. In: Intelligent robots and systems (IROS), 2011 IEEE/RSJ International Conference on, pp 973-979

Zecca M, Micera S, Carrozza MC, Dario P (2002) Control of multifunctional prosthetic hands by processing the electromyographic signal. Crit Rev Biomed Eng 30(4-6):459-485 